\section{Factors affecting the processing of sequentially presented form parts ${ }^{1,2}$}

WILLIAM D. SHONTZ, Systems and Research Center, Honeywell, Inc., St. Paul, Minn. 55113

An alternative to McFarland's (1965) model for the processing of visual form was tested. Form-part configuration, order of sequencing, and amount of change in part orientation within a sequence were investigated as factors affecting the processing of sequentially presented form parts. The results support McFarland's finding that the visual system takes longer to integrate a sequence of angles than a sequence of sides.

For obvious reasons, investigations of visual form perception have tended to concentrate on spatial rather than temporal factors. However, prominent theories of form perception have given temporal factors a central role. This has been done through the postulation of a scanning process.

Models such as Wiener's (1948) or Pitts \& McCullock's (1947) have postulated internal scanning mechanisms. The models of Hebb (1949) and Attneave (1954) have emphasized the importance of eye movements in scanning spatial patterns.

Both of these approaches raise serious methodological problems. In the one case, an internal scanning mechanism is postulated which, by definition, is not amenable to control. With the concept of an external mechanism, on the other hand, there is the problem of dealing with both voluntary and involuntary eye movements.

The present study was directed toward the question of the feasibility of investigating the processing of visual form by presenting the parts of a form in temporal sequence.

McFarland (1965) has proposed a model for the processing of visual form which emphasizes time as a variable. A central mechanism was postulated that performs operations of analysis and integration. According to the model, form is analyzed into a sequence of responses to line and to angle portions of retinal stimulation. Integration, then, is the result of a response to the product of this analysis. Thus, perception of form as simultaneous in time and joined in space is a result of these two operations-analysis and integration.

Fig. 1. Experimental conditions used by McFarland (1965).
To provide data in support of his model, McFarland presented parts of an equilateral triangle in temporal sequence. The outline of the triangle was luminous. Form parts presented were sides or angles configured as shown in Fig. 1. Each form part was luminated for $10 \mathrm{msec}$. The interpart intervals illustrated by gaps in the figures were varied using the method of limits. After each stimulus presentation, the $\mathrm{O}$ was asked to report whether the form's parts appeared simultaneous, overlapping, or successive in time. McFarland found that Os were more sensitive to sequencing when angles were used than when sides were used. That is, the threshold for simultaneity judgments was lower when angle parts were used.

Obviously, there is a great deal of commonality between the procedures used by McFarland and those used in much of the critical flicker fusion and apparent movement research. In fact, there exists in the apparent motion literature evidence to suggest that factors other than form-part configuration may account for results such as those McFarland obtained.

Orlansky (1940) found that changes in the orientation of the contours of pairs of forms presented alternately affected sensitivity to sequencing. Orlansky's measure of performance incorporated an estimate of a simultaneity threshold. He found that sensitivity to sequencing was greater when differently oriented pairs of the same forms were used than when like-oriented form pairs were used. Pollack (1966) confirmed this finding.

Thus, it appears that two major variables may account for changes in sensitivity to sequencing when form parts are presented sequentially, viz, form-part configuration and change in form-part orientation as the

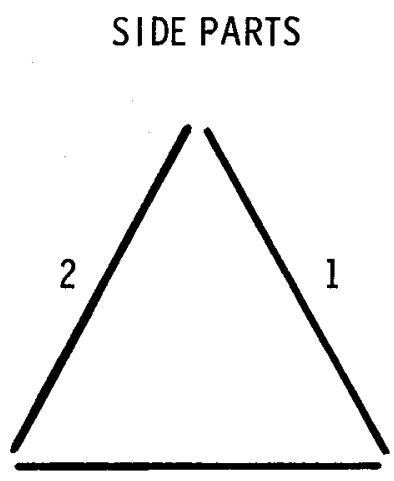

3 form parts are presented consecutively with a sequence. Let us assume that the sequence affects sensitivity to sequencing so that the greater the amount of change in orientation, the greater the sensitivity. If this assumption is correct, then McFarland's findings can be accounted for in these terms.

The present study was designed to test the adequacy of predictions based upon form-part configuration vs amount of change in form-part orientation as major variables affecting changes in sensitivity to sequencing. A luminous outline square was used as the stimulus pattern to permit manipulation of the orientation variable. Form-part configurations and orders of sequencing used are shown in Fig. 2.

With reference to Fig. 2, then, the amount of change in orientation of form parts presented consecutively can be determined for each experimental condition. Considering Experimental Conditions $A$ and $B$ first, it can be seen that, for both side and angle parts, a 90-deg change in orientation occurs with each consecutively presented form part. In Experimental Condition D, the maximum amount of change among the four conditions occurs. A 180-deg change occurs between Angles 1 and 3 and Angles 2 and 4. Added to this is the 90-deg change between Angles 3 and 2. On the other hand, the smallest amount of change in orientation takes place in Experimental Condition $\mathrm{C}$. There is no change in orientation between Sides 1 and 3 and between Sides 2 and 4 . The only change is a single 90-deg change between Sides 3 and 2.

Thus, if amount of change in orientation of consecutively presented form parts is a major factor affecting sensitivity to sequencing, the following relationship among the experimental conditions should obtain. Os should be most sensitive to sequencing under Conditions $A$ and $B$ and least sensitive under Condition $C$.

If McFarland's proposed model is

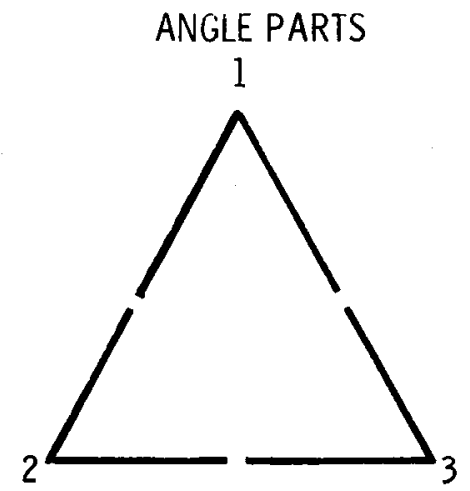


Fig. 2. Experimental conditions used in the present study.

applied to the conditions of the present study, then a different set of predictions regarding sensitivity to sequencing is obtained. It is McFarland's contention that line parts and angle parts are processed differentially. Therefore, the experimental conditions should be ordered as rollows: Os should be most sensitive to sequencing under Conditions $D$ and $B$, where angles are used. There would be no basis for predicting differences in sensitivity between these two conditions. Os should be least sensitive to sequencing under Conditions $A$ and $C$, where sides are used. Again, there would be no basis for differentiating between these latter conditions in terms of sensitivity to sequencing.

\section{METHOD}

Sixteen male undergraduates at lowa State University served as paid Ss. All had normal visual acuity, with or without correction, and normal color vision.

Eight $2 \times 2$ in. General Electric electroluminescent panels, Type $F$, were used to luminate the outline of the square. The panels were appropriately arranged and masked to produce the stimulus form. These EL panels have rise and fall times near $1 \mathrm{msec}$, so their response approximates a square wave. The panels were driven at 400 cycles and $100 \mathrm{~V}$. Excitation of the panels was controlled through Massey Dickinson behavioral programming modules. Stimulus presentation was viewed under dark-adapted conditions.

The procedure used in the present study was designed to permit the collection and analysis of psychophysical data on sensitivity to sequencing in accordance with the signal detection theory model. A rating-response technique employing a six-category rating scale was used (Green \& Swets, 1966). On each experimental trial, the probability of presenting the form parts, either sequentially or simultaneously, was 0.5 . After each presentation of the stimulus, 0 responded with one of the six scale category labels: (1) sequential-very sure, (2) sequential-fairly sure, (3) sequential-guess, (4) simultaneousguess, (5) simultaneous-fairly sure, (6) simultaneous-very sure.

The Os were given no specific information about the nature of the form parts or the order of sequencing.

Each $O$ completed three training sessions and three data collection sessions. The interpart interval was continually reduced during the training sessions as 0 became more proficient in discriminating between

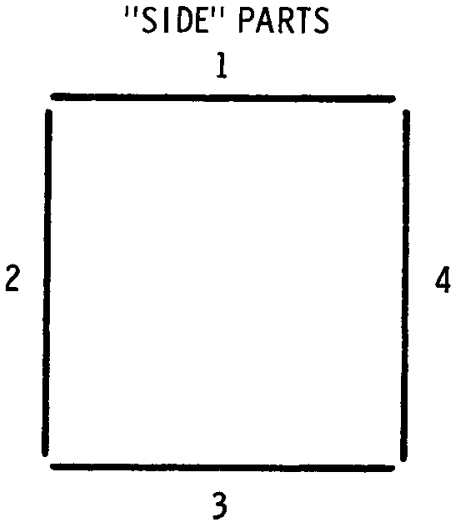

ORDER OF SEQUENCING:

1, 2, 3, 4 (EXPERIMENTAL CONDITION "A")

\section{1, 3, 2, 4 (EXPERIMENTAL CONDITION "C")}

modes of presentation. An attempt was made to determine the interpart interval for each $\mathbf{O}$ that would produce an average hit rate of about $70 \%$. This procedure was used to maximize any difference in sensitivity which might exist across experimental conditions.

Once an interpart interval was determined for an $O$ during training, that interval was maintained for him throughout all experimental conditions in all data collection sessions. Thus, interpart interval varied across $O s$, but this variation was not confounded with experimental conditions.

A Latin-square design with replicated square was used to determine the order in which experimental conditions were presented to Os. All experimental conditions were presented in each session. The data were summed across the three data-collection sessions to provide 300 trials per condition per $O$.

\section{RESULTS AND DISCUSSION}

Receiver operating characteristic, or ROC, functions were calculated for each 0 under each experimental condition resulting in a total of 64 such functions. The ROC functions were plotted on double probability paper to determine if $d^{\prime}$ could be used as a measure of sensitivity. Inspection of the functions indicated that in some cases a straight line appeared a reasonable fit of the data, but for others the best fit appeared decidedly curvilinear. On this basis, it was determined that the assumptions underlying the use of a $\mathrm{d}^{\prime}$ measure could not be met for all the data. Therefore, a distribution-free measure of sensitivity-the area under the ROC curve-was chosen as most appropriate for

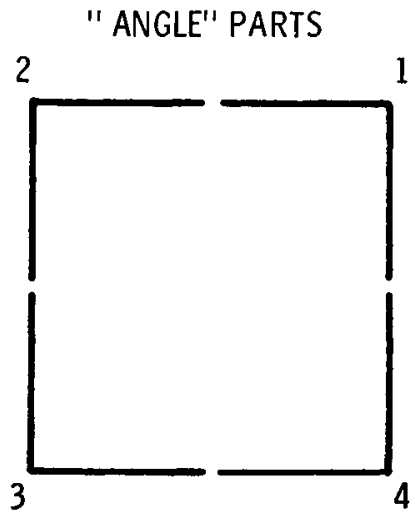

ORDER OF SEQUENCING:

$1,2,3,4$ (EXPERIMENTAL CONDITION 'B")

\section{1, 3, 2, 4 (EXPERIMENTAL CONDITION "D")}

the data. A summary version of the ROC functions is shown in Fig. 3. Data points were averaged across Os within experimental conditions. The effect of form-part configuration is apparent here. If amount of change in orientation had been a viable factor, the curves for Conditions $f$ and $B$ should have been superimposed midway between the curves for Conditions $C$ and $D$.

An area value was computed for each 0 under each condition. A split plot analysis of variance was performed using these 64 values. The effect of form-part configuration was significant $(F=37.72$, $\mathrm{df}=1 / 12, \quad \mathrm{p}<0.0005)$. The effect of

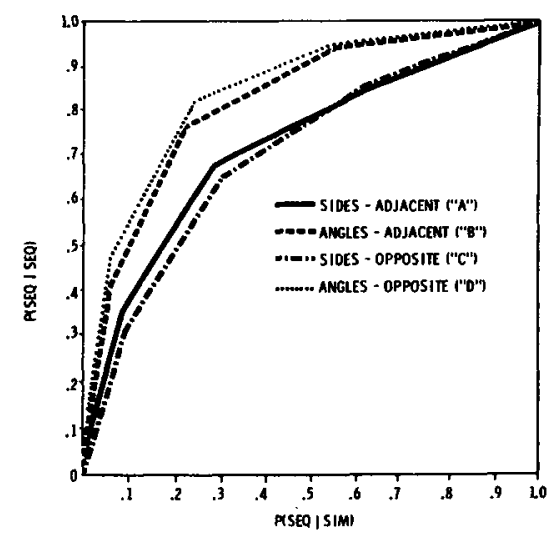

Fig. 3. Average ROC curves obtained from the four experimental conditions. (The $y$ axis may be read as "the probability of responding 'sequential' given sequential presentation of form parts." The $x$ axis may be read as "the probability of responding 'sequential' given simultaneous presentation of form parts.") 
amount of change in orientation was represented by the Configuration by Sequence interaction. It is through the different combinations of form-part configuration and sequence of part presentation that the effect of change in orientation would obtain. This variable did not have a significant effect on performance $(F=3.93, \mathrm{df}=1 / 12)$. Thus the results confirm, with a different form involving angles of different degree, McFarland's finding that the visual system takes longer to integrate a sequence of angles than a sequence of sides.

\section{REFERENCES}

ATTNEAVE, F. Some informational aspects of visual perception. Psychological Review, 1954, 64, 183-193.

GREEN, D. M., \& SWETS, J. A. Signal detection theory and psychophysics. New York: Wiley, 1966.

HEBB, D. O. The organization of behavior. New York: Wiley, 1949.
McFARLAND, J. H. Sequential part presentation: A method of studying visual form perception. British Journal of Psychology, 1965, 56, 493-446.

ORLANSKY, J. The effect of similarity and difference in form on apparent movement Archives of Psychology, 1940, 35, No. 246.

PITTS, W., \& McCULLOCH, W. S. How we know universals: The perception of auditory and visual forms. Bulletin of Mathematica Biophysics, 1947, 9, 127-147.

POLLACK, R. H. Effects of figure-ground contrast and contour orientation on the temporal range of apparent movement. Psychonomic Science, 1966, 4, 401-402.

WIENER, N. Cybernetics. New York: Wiley, 1948.

\section{NOTES}

1. The research reported herein was conducted while the author was the recipient of an IBM fellowship at Iowa State University.

2. Presented as a paper at the Ninth Annual Meeting of the Psychonomic Society, St. Louis, Missouri, October 31, November 1, 2, 1968.

\section{Comment on Cross and Cross's "The misperception of rotary motion"}

\section{R. P. POWER, The Queen's University of Belfast, Belfast, Northern Ireland}

Cross and Cross have proposed that apparent reversal of rotary motion in depth $(A R)$ is a function of strength and type of misleading depth cues, but are concerned that this does not account for voluntary control of frequency of $A R$ and increase in $A R$ over time. It is claimed that both effects are due to explicit or implicit instructions.

Cross \& Cross (1969) have proposed that apparent reversal of rotary motion in depth (AR) occurs as a function of the strength and number of misleading depth cues, a view supported by other investigations (Power, 1966). They are concerned, however, about two facts that they say their theory cannot explain. These are (1) the increase in ARs reported by Ss over time, and (2) the apparent ability of that fulfilling the instructions increases the probability of a similar response being made on a future occasion, Power had independent groups of Ss observe an Ames window under two conditions of instructions, to report the occurrence of ARs and to report the occurrence of rotation through the frontoparallel plane (i.e., a nonreversal). The experiment was repeated with an elliptical shape. It was found (Power, 1966) that in each case there was no significant difference in number of ARs on Trial 1 of four trials for the two sets of instructions, but that by Trial 2, in the case of the trapezoidal window, and Trial 4, in the case of the ellipse, there was a significant difference in frequency of AR. Thus the hypothesis was confirmed, and it may be concluded that there is no tendency for the effect to increase as a function of viewing time, but rather that there is a tendency to report an increase in frequency of $A R$ as a function of reporting ARs.

It is difficult to know to what extent Ss can control rate of AR. Ss often find it difficult to be sure whether they perceived rotation or oscillation and it may be that if they are endeavoring to control perceived direction they will interpret a doubtful AR as an AR or rotation, depending on their intention at the time. Although most people who have frequently experienced the illusion believe that they can exert voluntary control, it would be difficult to interpret unequivocally the results of an experiment that claimed to show this, since the demand characteristics of the situation might well lead the Ss to report a fewer or greater number of ARs independently of their perception. From informal observations it seems likely that such voluntary control as occurs is exerted by fixating on some point on the object and following it carefully, thus gaining information to true direction of rotation, or by letting the gaze relax and observing the object as a whole. If this is the case, then what seems to be voluntary control is in fact use of implicit instructions to fixate various parts of the stimulus object.

Thus, the type of explanation offered by Cross and Cross is not in difficulty. If the above analyses are correct, the facts that are not explained are a function of extra variables and do not embarrass a theory based on features of the stimulus.

\section{REFERENCES}

CROSS, J., \& CROSS, J. The misperception of rotary motion. Perception \& Psychophysics, $1969,5,94-96$.

POWER, R. P. The effect of instructions on the apparent reversal of rotary motion in depth. Quarterly Journal of Experimental Psychology, 1965, 17, 346-350.

POWER, R. P. Studies in the apparent reversal of rotary motion in depth. Unpublished PhD thesis, University of Sydney, 1966. 